\title{
Outbreak evaluation of highly pathogenic avian influenza in Bangladesh
}

\author{
M. Giasuddin ${ }^{1 *}$, M.E.Haque ${ }^{2}$, A.H.M.Kamal ${ }^{2}$, M.R. Islam², A. Jahangir ${ }^{1}$, E.H. Chowdhury ${ }^{2}$ \\ M.J.F.A.Taimur ${ }^{1}$ and M. Hafizur Rahman ${ }^{1}$ \\ ${ }^{1}$ Animal Health Research Division, Bangladesh Livestock Research Institute, Savar, Dhaka. \\ ${ }^{2}$ Department of Pathology, Faculty of Veterinary Science, Bangladesh Agricultural University, \\ Mymensingh \\ *Corresponding author: mgias04@yahoo.com
}

\begin{abstract}
Bangladesh first experienced outbreaks of highly pathogenic avian influenza (HPAI) subtype H5N1 in poultry 2007 and by December 2012 a total of five hundred fifty six (556) outbreaks have been reported of which four hundred ninety nine (499) outbreaks occur in commercial poultry farm as against only fifty seven (57) in backyard poultry chicken. The virus appeared to be a deadly pathogen causing a total of six hundred eight (608) human cases with three hundred fifty nine (359) deaths in the world. In Bangladesh seven (7) human cases have been reported with a singular mortality of a child acquiring the infection from household poultry. There had been six epidemic waves of AI outbreaks in Bangladesh since March 2007 and other new waves seem to have started. From the six year's incidence analysis it was found that higher number of outbreaks occurred in the month of February followed by March. The outbreak started from the middle to late winter and continued up to summer. The phylogenetic analysis of viruses isolated till 2010 revealed only one clade 2.2 virus circulating in Bangladesh. But from 2011 two new clades 2.3.2 and 2.3.4 viruses have been introduced. In 2012, it was observed that Clade 2.2 viruses that was in circulation since 2007 were replaced by 2.3.2.1 viruses. Extensive backyard poultry including a large number of ducks, dense human population, and economic dependence of poor people on poultry with low awareness about risk of infection, live bird trading and poor bio-security were critical factors in the spread of avian influenza infection that posses key challenge in rapid containment. Because of the complex situation in poultry production and marketing system, attempts to control this disease through stamping out and bio-security measures have apparently failed in Bangladesh.
\end{abstract}

(Key words: Avian influenza, Pathogenic, RT-PCR, Phylogenetic)

\section{Introduction}

Since the emergence of H5N1, HPAI panzootic in late 2003, the disease has spread in Asia as well as all over the world including sixty three (63) countries of five (5) continents. There have been a considerable efforts made by the countries, international and regional organizations to control the disease. However, the disease is still continuing to cause economic losses as well as 
public health concerns. As Bangladesh has a long border with India and Myanmar it was considered as a high risk country for introduction of HPAI following outbreak in early 2006 in the neighboring countries. The country first experienced HPAI in early 2007 and the National Reference Laboratory for Avian Influenza (NRL-AI) at Bangladesh Livestock Research Institute (BLRI) has diagnosed and confirmed the presence of H5 near the capital Dhaka. The disease gradually spread to at least fifty one (51) of the country's sixty four (64) districts (Giasuddin, et al. 2009). Six human cases of avian influenza were identified in Bangladesh and were reported to WHO in (2011). Avian Influenza viruses (AIV) usually in low pathogenic (LPAI) form are frequently isolated from wild birds specially waterfowl (Alexander, 1982) that act as a reservoir and serves as the source of infection In domestic poultry. The persistence of the virus in poultry over a wide geographic area may increase the risk that a mutant virus might evolve to initiate a human pandemic. Bangladesh has a complex structure of poultry production with large and small commercial farm and extensive backyard poultry including a large number of ducks. The economic dependence of poor people on poultry with low awareness about risk of infection, lo bio-security in small scale commercial poultry, live bird trading are found critical factors in the spread of infection over the last six waves (Giasuddin, et al. 2011). The present paper reported the findings of ongoing passive as well as some active virological surveillance of AIV in Bangladesh.

\section{Materials and Methods}

\section{Clinical samples}

Clinical samples including tracheal tissue were collected from the avian influenza suspected poultry by the Field Disease Investigation Laboratory (FDIL) and Central Disease Investigation Laboratory (CDIL) of Department of Livestock Services (DLS) from different areas of the country. The samples were then submitted to National Reference Laboratory for Avian Influenza (NRL-AI) for confirmatory diagnosis of Avian Influenza viruses (AIV). The study was conducted over the period from March 2007-December 2012 during which a total 619 samples were received and tested. 


\section{Surveillance samples}

The work was conducted during 2007-2010 during which a total of 1500 cloacal swab and 500 serum samples from native ducks and 2050 fecal samples from wild migratory birds were collected randomly from different areas of Bangladesh and transported to NRL-AI.

\section{Sample processing}

RNA was extracted from the clinical samples and real time RT-PCR was performed. Surveillance samples were inoculated in embryonated chicken eggs. Allantoic fluid collected from embrocated eggs and tested by haemagglutination test followed by either RT-PCR or real time RT-PCR for identification of influenza A subtype.

\section{Phylogenetic analysis}

Nucleotide sequence of partial HA gene of selected samples were subjected to multiple alignment with the corresponding sequence of other HPAI strains of different countries available in the Gene Bank. Phylogenetic trees were developed and genetic clades of the viruses were identified.

\section{Results and Discussion}

During the early 2007, there were some reports of unusual mortality in commercial poultry farms from different parts of the country (Giasuddin, et al. 2011). In March 2007, NRL-AI first received five suspected samples from CDIL, Dhaka which were detected to be H5-AI virus by RT-PCR and the result was submitted to the Government of Bangladesh. Since then the disease outbreaks were reported every year (Giasuddin, et al. 2011). Till December 2012, 556 outbreaks were recorded of which 499 were in commercial chickens and 57 in backyard chicken. There have been six epidemic waves of AI outbreaks in Bangladesh since March 2007. From the last six wave's analysis, it is evident that the incidence of avian influenza starting in the winter and continued to the summer. The rate of infections declined with none or a few outbreaks in the monsoon (Fig. 1). 


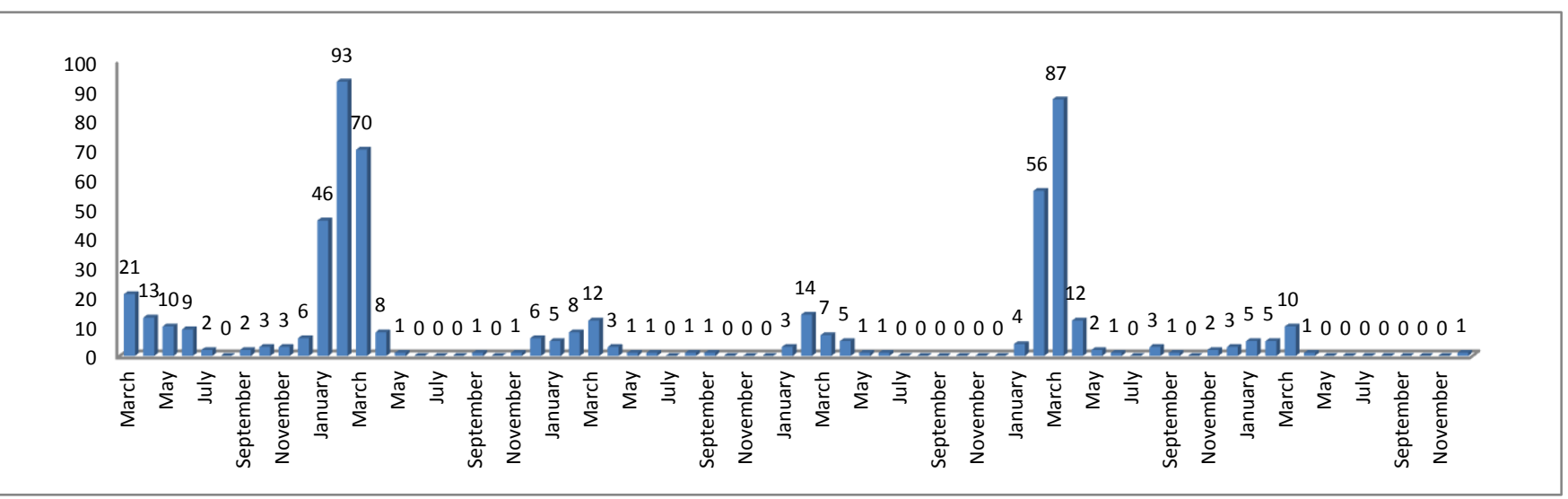

Fig: 1. Temporal distribution of HPAI outbreaks in Bangladesh.

Outbreaks of HPAI in Bangladesh were reported mostly from commercial poultry farms and more specifically in small scale layer farms. The higher incidence of outbreak in layer farms was due to the longer life span of the birds providing the opportunity for the birds, farm personnel and equipment to come in contact with the contaminated materials. Unorganized commercial poultry farms and wet marker with poor biosecurity measures seemed to be the major factors in maintaining the disease during the last six years in this country. Up to 2010, the disease was reported mostly from chicken and a small number of incidences from crows, suspected to consume chickens died of avian influenza (Giasuddin, et al. 2011). But from 2011, deaths due to HPAI infection were reported from Quails and ducks (Islam, et al. 2012).

In Bangladesh, most of the poultry farms are being located near the city areas provided infection of AIV in high number in such poultry dense areas. The source might be also large in live bird markets (wet market) where poultry are transported from all over the

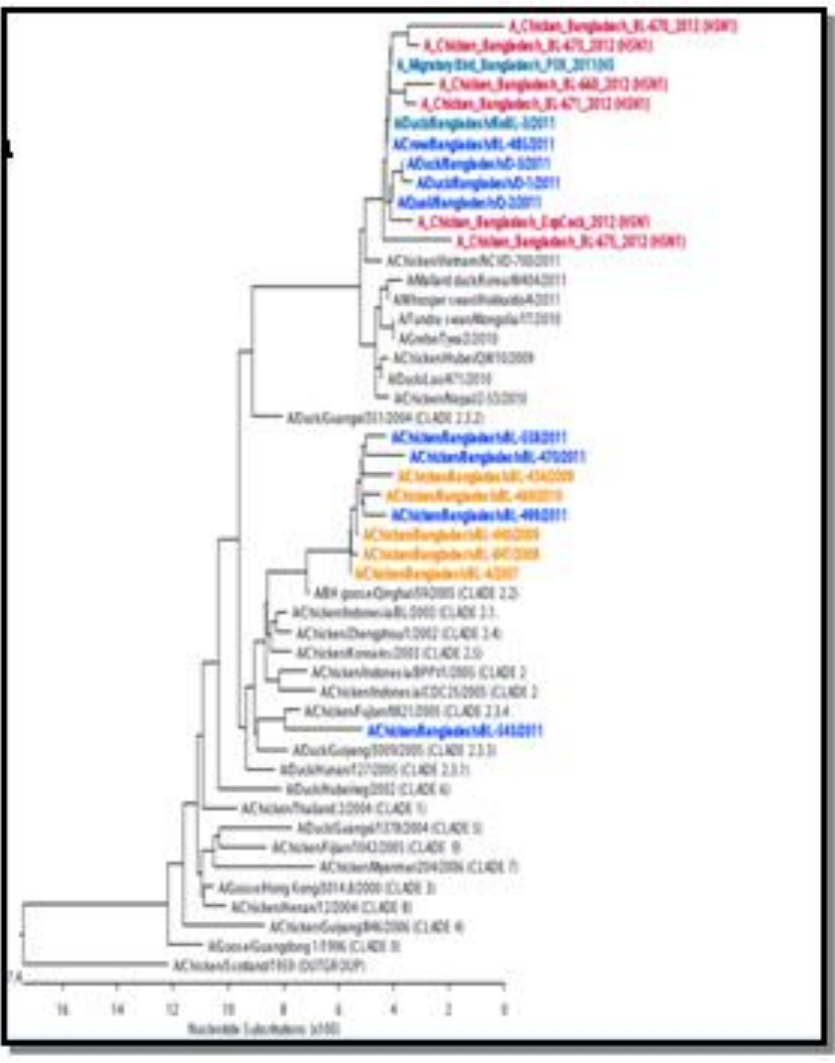

Fig. 2. Phylogenetic analysis of Bangladeshi $\mathrm{H} 5 \mathrm{~N} 1$ isolates country. 
The phylogenetic analysis of Bangladeshi isolates of HPAI collected from chickens during the period from 2007 to 2010 demonstrated that Bangladeshi isolates clustered with those from middle China, South mid Asia, Middle-East, Europe and Africa popularly known as Qinghai Lineage or Euro-Asia Africa lineage belonging to a single genetic clade 2.2. But in 2011 viruses of two new clades 2.3.2 and 2.3.4 (Fig. 2) were also introduced that co-circulated with the previous one (Islam, et al. 2012).

Representative samples of 2012 HPAI infections from different areas of Bangladesh were also used for molecular characterization and phylogenetic analysis. Phylogenetic analysis of these viruses revealed that all $\mathrm{H} 5 \mathrm{~N} 1$ viruses belonged to the 2.3.2.1 clade with a similarity ranging between $97.4 \%$ and $100 \%$ (data not shown). They clustered with $\mathrm{H} 5 \mathrm{~N} 1$ viruses previously collected in Bangladesh and with sequences from Nepal (2011-2012), Myanmar (2010-2011), and India (2011-2012) (Islam et al. 2012). It was observed that clade 2.2 viruses are being replaced by clades 2.3.2.1 virus that has been in circulation since 2007 .

To explore the potential reservoir hosts, 1500 cloacal swab and 500 sera samples from indigenous ducks and 2050 fecal samples from wild migratory birds were collected. Out of 1500 cloacal swabs, 17 samples (1.13\%) were found haemagglutination positive and from them three viruses were confirmed as influenza A positive by RT-PCR. On the other hand, 32\% duck was found sero-positive to influenza A virus. From migratory birds, four (4) samples were found haemagglutination positive and of these two were confirmed as H5N1. Like other countries in this region, ducks seems to be one of the major contributors to HPAI outbreaks in backyard chickens in Bangladesh.

\section{Conclusion}

Since 2011 there has been introduction of two new clades of HPAI viruses, clade2.3.2.1 and 2.3.4 into Bangladesh in addition to clade 2.2 viruses, but in 2012 it was observed that clade 2.2 viruses are being replaced by 2.3.2.1 virus that were in circulation since 2007 .

\section{References}

Alexander, D.J. 1982. Avian influenza-recent developments, Veterinary Bulletin 52: 341-359. 
Giasuddin, M., Taimur, M.J.F.A., Jahangir, A., Al-Mamun, M. and Samad, M.A. 2007. Monitoring of avian influenza: status in Bangladesh. Proceedings of the $5^{\text {th }}$ International Poultry Show and Seminar held on 1-3 March, Bangladesh -China Friendship Conference Center, Dhaka, Bangladesh, pp. 102-108.

Giasuddin, M., Samad, M.A., Khan, M.S., Monoura, P., Al-Faruque, M.H. Saha, S.S. and Sultana, S. 2009. Outbreak and frequency of circulating strains of avian influenza virus in Bangladesh from 2007-2008. Proceedings of the $6^{\text {th }}$ International Poultry Show and Seminar held on March 5-7, Bangladesh -China Friendship Conference Center, Dhaka, Bangladesh, pp. 111-5.

Giasuddin, M., Jahangir, A., Samad, M.A., Monoura, P., Al-Faruque, M.H. and Taimur, M.J.F.A. 2011. Outbreak and frequency of circulating strains of avian influenza virus in Bangladesh from 2007-2008. Proceedings of the $7^{\text {th }}$ International Poultry Show and Seminar held on March 5-7, Bangladesh-China Friendship Conference Center, Dhaka, Bangladesh, pp.63-68.

Islam. M.R., Haque, M.E., Giasuddin, M.,Chowdhury, E.H., Samad, M.A., Parvin, R., Nooruzzaman, M., Rahman, M.M. and Monoura, P. 2012. New Introduction of Clade2.3.2.1 Avian Influenza Virus (H5N1) into Bangladesh. Transboundary and Emerg. Dis., 59: 460-463.

WHO 2011. www.who.Int/csr/disease/avian_influenza/country/cases_table_2008_09_10_11/ index.htt. 